\title{
Editorial-advances in nanomanufacturing: process repeatability, scalability and affordability for nanomaterials and manufacturing
}

\author{
O. Arda Vanli • Iris V. Rivero • Ben Wang
}

Published online: 28 November 2012

(C) Springer-Verlag London 2012

Nanomanufacturing comprises the technologies for designing, producing, manipulating, and assembling devices and systems that have features in the nanometer scale. The past few decades have seen substantial progress in the research of nanomaterials and processes development. However, many challenges still remain in the areas of repeatability and scalability of nanomanufacturing processes. Without process repeatability, production scale-up, and a strong market pull, nanomaterials will remain a benchtop exercise without real commercial impact or societal benefits. There is a profound need for innovation-driven and cost-effective translational technologies that will address these challenges. This special issue was an attempt to gather recent developments that address the issues of repeatability and scalability in nanomanufacturing.

This special issue contains six papers addressing the areas of process modeling, monitoring, control, and scale-up of nanomanufacturing processes. The first paper, Chen et al., proposes a new method for making backlighting devices from carbon nanotube buckypaper using laser irradiation. The authors apply a fractional factorial experimental design and a response surface model to rapidly search the feasible laser parameter settings for processing buckypaper field emitters and

\footnotetext{
O. A. Vanli $(\bowtie)$

Florida State University, Tallahassee, FL, USA

e-mail: avanli@fsu.edu

I. V. Rivero

Iowa State University, Ames, IA, USA

e-mail: rivero@iastate.edu

B. Wang

Georgia Institute of Technology, Atlanta, GA, USA

e-mail: ben.wang@gatech.edu
}

improving field emission properties within few experimental runs. The second paper, Desai et al., proposes a new direct inkjet writing method to deposit enriched single-wall nanotubes for fabricating flexible thin film transistors. The authors show that the direct writing technique has the benefit of accurately controlling the deposition and high reproducibility in printing nanotubes over large areas. In the third paper, Abuhimd et al. investigate a new approach to control the output repeatability of the chemical vapor deposition process when growing single-wall carbon nanotubes. The method relies on applying statistically designed experiments to determine an effective combination of the process variables, such as hydrocarbon gas flow rate, chamber temperature, and chamber pressure, for the precise control of nanotube lengths.

In the fourth paper of the special issue, Tolle and Martin study an optimization-based methodology in order to identify network process-structure-property relationships in multiphase polymeric materials. A computationally efficient decomposition approach is developed in order to extract important patterns of the material structure from X-ray and neutron scattering imaging data with minimal a priori information. In the fifth paper, Tseng et al. presents a continuous precipitation method for the fabrication of ceria nanoparticles using a new micromixer process. It is shown that the approach has a higher throughput, which is important for scaling up, and that it is capable of producing more uniform porous film structures than standard batch mixing process. In the sixth and final paper, Qin et al. discuss a large-scale process optimization method to be used for planning a focused ion beam process when performing threedimensional patterning. It is shown, using the proposed process planning method, that significant improvement in computational time and economical cost can be achieved in the beam scanning process. 
We are grateful to the fine contributions of the authors who submitted papers. The papers were processed through two rounds of peer reviews. In each round, the papers were evaluated by two anonymous reviewers. Here, we would like to acknowledge the assistance we received from the reviewers whose input significantly improved the quality of the papers. Finally, we wish to express our sincere gratitude to Professor B. J. Davies, Editor-in-Chief of the International Journal of Advanced Manufacturing Technology (IJAMT), for giving us the opportunity to develop this issue, and to Claudia Kehl from the IJAMT editorial office, for her valuable guidance throughout the publication process. 